\title{
Bessel Terahertz Pulses from Superluminal Laser Plasma Filaments
}

\author{
Zhelin Zhang, ${ }^{1,2,3}$ Jiayang Zhang, ${ }^{2,3}$ Yanping Chen $\mathbb{D}^{2,3}$ Tianhao Xia, $^{2,3}$ Linzheng Wang, ${ }^{2,3}$ \\ Bonan Han, ${ }^{2,3}$ Feng He, ${ }^{2,3}$ Zhengming Sheng, ${ }^{1,2,3,4}$ and Jie Zhang ${ }^{1,2,3}$ \\ ${ }^{1}$ Tsung-Dao lee Institute, Shanghai Jiao Tong University, Shanghai 200240, China \\ ${ }^{2}$ Key Laboratory for Laser Plasmas (Ministry of Education), School of Physics and Astronomy, Shanghai Jiao Tong University, \\ Shanghai 200240, China \\ ${ }^{3}$ Collaborative Innovation Center of IFSA, Shanghai Jiao Tong University, Shanghai 200240, China \\ ${ }^{4}$ Department of Physics, SUPA, University of Strathclyde, Glasgow G4 ONG, UK
}

Correspondence should be addressed to Yanping Chen; yanping.chen@sjtu.edu.cn

Zhelin Zhang and Jiayang Zhang contributed equally to this work.

Received 14 December 2021; Accepted 4 February 2022; Published 2 March 2022

Copyright (C) 2022 Zhelin Zhang et al. Exclusive Licensee Xi'an Institute of Optics and Precision Mechanics. Distributed under a Creative Commons Attribution License (CC BY 4.0).

Terahertz radiation with a Bessel beam profile is demonstrated experimentally from a two-color laser filament in air, which is induced by tailored femtosecond laser pulses with an axicon. The temporal and spatial distributions of Bessel rings of the terahertz radiation are retrieved after being collected in the far field. A theoretical model is proposed, which suggests that such Bessel terahertz pulses are produced due to the combined effects of the inhomogeneous superluminal filament structure and the phase change of the two-color laser components inside the plasma channel. These two effects lead to wavefront crossover and constructive/destructive interference of terahertz radiation from different plasma sources along the laser filament, respectively. Compared with other methods, our technique can support the generation of Bessel pulses with broad spectral bandwidth. Such Bessel pulses can propagate to the far field without significant spatial spreading, which shall provide new opportunities for terahertz applications.

\section{Introduction}

Bessel beams have received increasing attention due to their unique applications in optical imaging, optical micromanipulation, optical microprocessing, and optical communications [1-9]. In imaging systems, for instance, due to its special feature of propagation, Bessel beams can enhance the ability of 3D imaging [10]. Even though Bessel beam profiles have been well demonstrated in the optical region, it is still a challenge for the generation of terahertz Bessel beams with broad bandwidth, which can play a pivotal role in biomedical and security imaging, etc. [11, 12]. In the past two decades, the generation of terahertz Bessel beams has been demonstrated either by a V-shaped metamaterial [13] or by 3D-printed axicons [14]. Thus, terahertz Gaussian beams are converted to Bessel ones within the interfering region formed by these devices. However, due to the limita- tion by insufficient adaptive spectral bandwidth in the terahertz range as well as nonnegligible energy loss from absorption of these devices, the methods metioned above are not applicable for broadband terahertz radiation from laser filament sources. Since the beginning of this century, laser filaments excited by high-power femtosecond lasers have attracted lots of research interests due to their wide applications such as generation of broadband terahertz radiation, atmospheric sensing, air lasing, and laser-induced spark ignition [15-18]. Recently, it was demonstrated that the beam divergence of the conical terahertz radiation from laser plasmas can be modified by extending laser plasmas to a long laser filament, i.e., a longer plasma filament resulting in a conical terahertz radiation with a smaller divergence $[19,20]$. This suggests that the beam profile of the terahertz radiation from laser plasmas might be shaped by modifying the structure of laser plasma sources. 
In this work, we demonstrate the generation of a Bessellike terahertz pulse from a two-color laser filament whose ionization front travels faster than light in vacuum. A typical high-order Bessel beam profile of terahertz radiation is observed experimentally. Based on theoretical investigation, it is found that the generation of the Bessel terahertz pulse is due to the combined effects of the superluminal filament structures and the phase changing of the two-color laser components inside the plasma channel. The observed Bessel terahertz pulse with our two-color superluminal filament scheme can propagate to the far field, no longer limited in a short interfering region as usually found in other methods mentioned above. This work shows the possibility of beam shaping for terahertz waves by employing a suitable plasma filament for the first time.

\section{Materials and Methods}

In our experiment, a $16 \mathrm{~mJ}, 40 \mathrm{fs}$, and $800 \mathrm{~nm}$ laser pulse with a beam diameter of $50 \mathrm{~mm}$ is focused by a $50 \mathrm{~cm}$ focal long planoconvex lens. Before the laser beam undergoes filamentation process, it is intercepted sequentially by an axicon with a wedge angle of $4^{\circ}$ and a $\beta$-barium borate (BBO) crystal of $0.2 \mathrm{~mm}$ thickness and a dual waveplate, as sketched in Figure 1. After the generation of the second harmonic with the $\mathrm{BBO}$ crystal, the polarization of the $800 \mathrm{~nm}$ wave is tuned to be parallel to its second harmonic by the dual waveplate. Therefore the generated $\mathrm{THz}$ radiation will has the same polarization as the laser pulses. Then, a $55 \mathrm{~mm}$ long plasma filament, excited by the $800 \mathrm{~nm}$ fundamental wave and its second harmonic, is formed just after the dual waveplate. Terahertz radiation from this plasma filament is collected by a pair of 2-inch off-axis parabolic (OAP) mirrors with a focal length of $10 \mathrm{~cm}$ and detected by electrooptic sampling technique $[20,21]$. A $0.5 \mathrm{~mm}$ thick silicon wafer is placed inside the OAP pairs to block the two-color laser beam, whose beam profile after filamentation is like a ring. The mechanism of $\mathrm{THz}$ radiation with two-color lasers in the present work is due to the ionization currents as discussed in many previous studies $[20,22]$. The introduction of the axicon here is to modify the propagation velocity of the ionization front of the laser filament, eventually responsible for Bessel terahertz beam generation. Previously, it was demonstrated that terahertz radiation from a superluminal ionization front created by an axicon-focused one-color femtosecond laser pulse has a conical radiation profile [23].

We diagnosed the waveform of the terahertz radiation from the superluminal laser filament as a function of its emission angle by changing the diameter of the aperture for the metal iris located after the filament (detailed in Figure 1), as shown in Figure 2(a). It is notable that the amplitudes of the terahertz waveforms first increase and then fluctuate slowly (shown in dashed line) as the diameter of the iris aperture $d$ is enlarged gradually, while terahertz carrier-envelop phases stay almost unchanged. By subtracting the terahertz waveform obtained at a given iris diameter $d$ by the one measured with iris aperture of $(d-\Delta d, \Delta d=2$ $\mathrm{mm}$ ), we can retrieve radially terahertz waveforms in Figure 2(b) [24]. Here, the $z$ axis corresponds to the laser

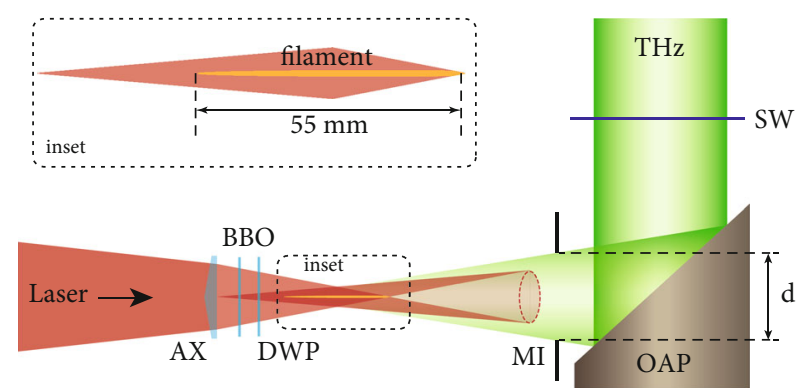

FIgURE 1: Schematic diagram of the experimental setup. The diameter of the adjustable metal iris is defined as $d$. AX: axicon; BBO: $\beta$-barium borate crystal; DWP: dual wave plate; MI: metal iris; OAP: off-axis parabolic; SW: silicon wafer. Inset: filament in the spindle region.

propagation axis, whose origin $(z=0)$ is defined at the end of the filament. The $r$ axis corresponds to the radial direction which is perpendicular to the $z$ axis. In this way, the superluminal filament is located from $z=-55 \mathrm{~mm}$ (the beginning of the filament) to $z=0 \mathrm{~mm}$, and the iris is placed at $z=65$ $\mathrm{mm}$ with its maximum aperture radius of $20 \mathrm{~mm}$. In Figure 2(b), it is obvious that the terahertz radiation from the superluminal filament separates into three rings with different conical angles. In addition, the terahertz waveforms show a $\pi$-phase difference from one ring to its neighbor ring, which is in accordance with a typical high-order Bessel ring structure [25].

\section{Results and Discussion}

Due to the existence of the axicon, the plasma filament formed with our experimental setup is different from normal filaments studied before [26]. The ionization front of the filament propagates with a superluminal velocity owning to the unique geometry of the axicon [23]. Meanwhile, the laser beam is focused by a planoconvex lens before passing through this axicon. This leads to a nonuniform propagation velocity of the ionization front as well as a nonuniform distribution of the laser intensity along the filament, as shown in Figures 3(a) and 3(b), respectively. These are obtained according to the geometric relation with the beam diameter of $20 \mathrm{~mm}$ on the axicon surface and the refractive index of the axicon $n=1.45$, respectively. Figure 3(a) shows that the ionization front of the filament starts with the velocity of about $1.006 c$, and gradually increases to about $1.016 c$, where $c$ is the speed of light in vacuum. This phenomenon suggests that the wavefront of the terahertz radiation from the plasma source located at the leading part of the filament travels slower than the ionization front of the filament, i.e., resulting in the wavefront intercross of the terahertz radiation from different plasma sources along this filament. In Figure 3(b), the laser intensity distribution along the filament is spindle-shaped, strong in the middle and weak in both ends. This is reasonable because of the beam profile modification by the axicon, consistent with Ref. [27, 28]. The detailed calculation is given in the Supplemental Materials (available here). 


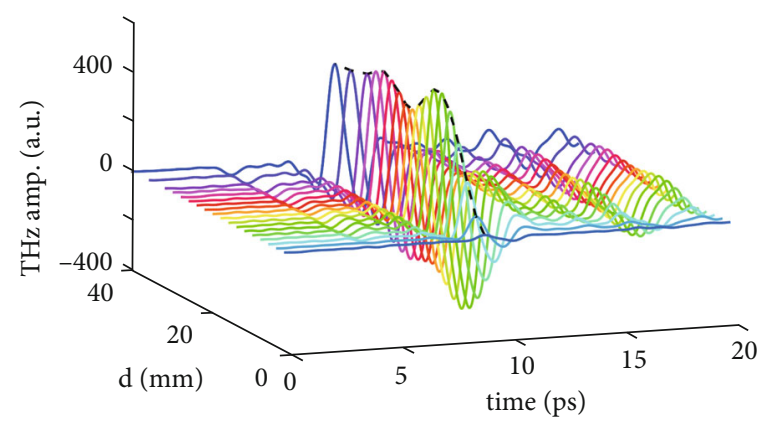

(a)

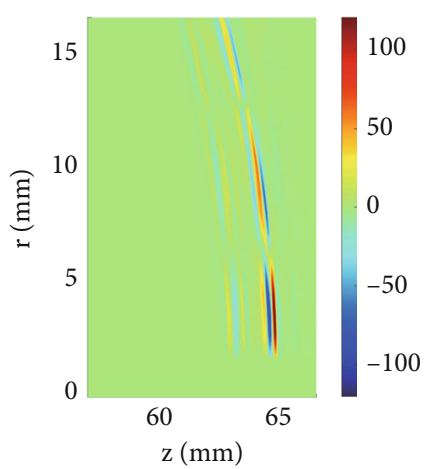

(b)

Figure 2: Experimental results. (a) Measured terahertz waveforms with different diameters of the metal iris $d$ (in Figure 1). The dashed line shows the peak evolution of the waveforms with $d$. (b) Spatial distribution of the terahertz waveforms obtained by subtracting each measured waveform by its former one with a smaller $d$ in (a).

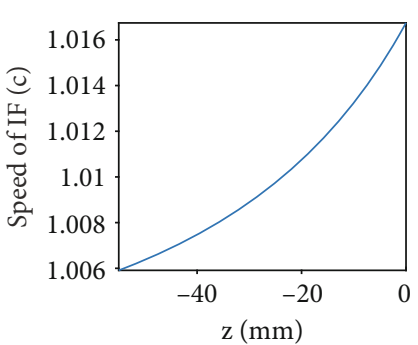

(a)

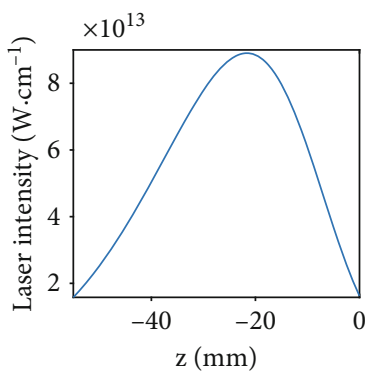

(b)
Figure 3: (a) Calculated propagation velocity of ionization front (IF) versus the longitudinal position of the filament. The position $z=0 \mathrm{~mm}$ represents the end of the filament. (b) Calculated laser intensity inside filament region along the longitudinal position of the filament.

Based on the features of the two-color superluminal plasma filament, we introduce a physical model called nonlinear-dipole-array model to describe terahertz radiation from this filament. First, each plasma point source (at position $z$ with length $\mathrm{d} z$ ) along the superluminal filament, whose plasma density is derived from the AmmosovDelone-Krainov ionization model, can be considered an oscillation dipole emitting a terahertz pulse [22, 29]. Such terahertz pulse is modified by the phase difference between two-color laser components $\phi(z)$ at different positions $z$ $[20,22]$, as expressed as $\tilde{E}(\omega, z, t) \sin [\phi(z)] \mathrm{d} z$, where $\omega$ is the angular frequency of terahertz radiation (see more details in the Supplemental Material). Taking into account the superluminal ionization front (i.e., the formation of the plasma point source) as well as the terahertz propagation effect $[20,30]$, the phase change of the terahertz radiation as it propagates to a given spatial position $\mathbf{r}(r, z)$ can be expressed as $\Delta \varphi=\omega\left(t-z / \alpha_{z} c\right)-k l(\mathbf{r}, z)$. Here, $\alpha_{z}$ is the ratio between the propagation velocity of the ionization front of the superluminal filament and the speed of light in vacuum (illustrated in Figure $3(\mathrm{a})$ ), $k=\omega / c$ is the corresponding wave number of the terahertz waves, and $l(\mathbf{r}, z)=\int_{s} n(\mathbf{r}) d \mathbf{r}$ is the effective optical path of the terahertz radiation, which is calculated through a path integration from the refractive index $n(\mathbf{r})$. In this way, the spatial distribution of terahertz radiation from a single-plasma point source can be calculated. Second, we suppose that all the terahertz plasma sources along the superluminal filament are independent of each other, i.e., the terahertz radiation from the leading part of the filament will not affect the process of laser plasma interactions in the tailing part of the filament. Thus, the beam profile of the terahertz radiation from a two-color superluminal filament can be expressed as a coherent superposition of the spatial distribution of the terahertz waves emitted from individual plasma sources distributed along the filament, i.e.,

$$
E^{\mathrm{THz}}(\omega, \mathbf{r}, t)=\int_{\text {fila. }} \tilde{E}(\omega, z, t) \sin [\phi(z)] \exp (\Delta \varphi) d z
$$

Compared with our previous work [20, 21], the ionization front (i.e., the formation of the plasma point source) in a superluminal laser filament travels faster than the wavefront of the emitted terahertz radiation from each plasma point source. Consequently, the terahertz wavefronts from different plasma point sources along the superluminal filament will meet and overtake. The subsequent constructive/ destructive interference with each other leads to a ring structure of a terahertz beam profile. Besides, due to different laser intensities distributed along the superluminal filament (as shown in Figure 3(b)), the resulting variance in plasma density and intensity of the generated terahertz radiation from each point source is also taken into account in the new model.

Using Equation (1), the radial distribution of the terahertz radiation from a $55 \mathrm{~mm}$ long superluminal laser filament is calculated, as shown in Figure 4(a). Our simulation with the above model can well reproduce the terahertz beam pattern of a high-order Bessel ring structure observed in the experiment, including a $\pi$-phase shift in the waveforms of terahertz radiation from adjacent rings. Figure 4(b) displays the propagation trajectory of the Bessel terahertz beam. Each vertical line in the figure represents the beam pattern of the Bessel beam at a specified location along the propagation axis. For instance, the observed Bessel beam profile in our experiment, diagnosed with the metal iris at $z=65 \mathrm{~mm}$, 


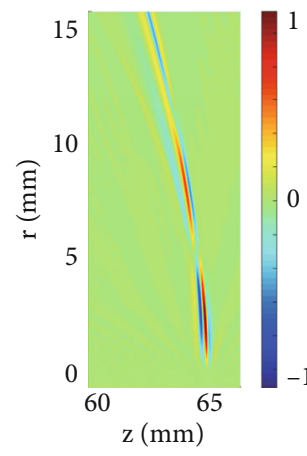

(a)

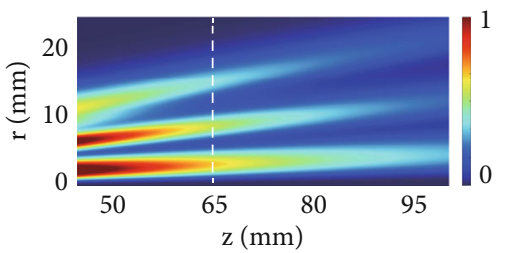

(b)

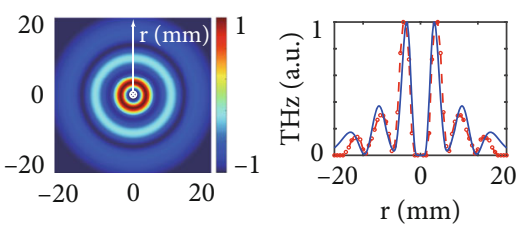

(c)

(d)

FIgURE 4: Simulation results and comparison with experimental measurements. (a) Spatial distribution of terahertz waveforms obtained numerically under experimental conditions as in Figure 2(b). (b) Power distribution of terahertz radiation while propagating. The dashed line represents the position of the metal iris. (c) Beam profile of Bessel terahertz radiation. (d) Intensity distribution of Bessel terahertz beam along transverse direction (blue solid line), compared with measured intensity distribution (experimental data in red circles) from Figure 2(b). Here, the measured distribution is the intensity integration along the $z$ axis of Figure 2(b), and the simulation curve comes from the cut section along the $r$-axis of (c).

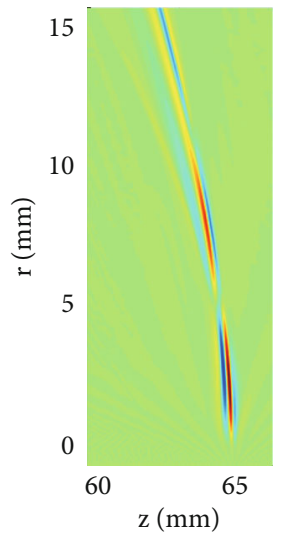

(a)

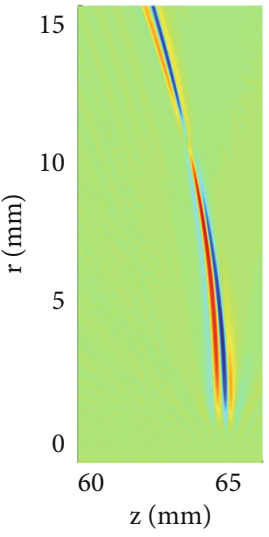

(b)

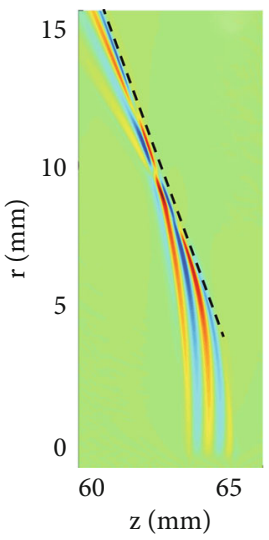

(c)

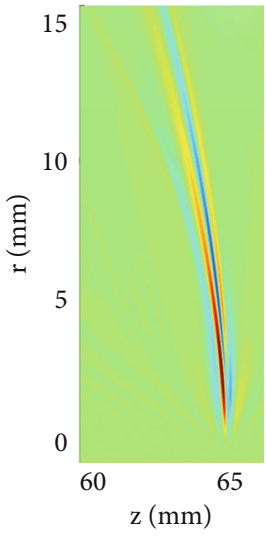

(d)

FIGURE 5: Simulated spatial distributions of terahertz radiations in different conditions. (a) Bessel beam from a $55 \mathrm{~mm}$ long superluminal filament (fit with our experiment conditions). (b) Bessel terahertz beam from a $42 \mathrm{~mm}$ long superluminal filament (lower laser energy). (c) Terahertz beam from a $55 \mathrm{~mm}$ long superluminal filament with uniform plasma density distribution and filamentation velocity (only axicon, no lens). (d) Traditional terahertz beam from a $55 \mathrm{~mm}$ long laser filament (no axicon and no superluminal phenomena).

corresponds to the beam pattern marked with a dashed line in Figure 4(b). The evolution of the beam pattern during propagation indicates that the terahertz radiation from our scheme remains its Bessel beam profile while propagating to the far field. Figure 4(c) shows this terahertz beam profile in a 2-D plane located at $z=65 \mathrm{~mm}$ (the dashed line in Figure 4(b)), which gives one a more intuitive sense of the three ring structures. Comparing the theoretical and the experimental data, the distribution and the relative strength of the three rings in the terahertz beam profile are in good agreement, as shown in Figure 4(d). Hence, our physical model well interprets the generation process of a Bessel terahertz pulse from a superluminal two-color laser filament.

In addition, we have studied the effects of the filament conditions on terahertz beam patterns numerically based upon our theory model presented above. First, we consider the effect of filament length. Figure 5(b) shows a two-ring beam pattern of terahertz radiation from a superluminal filament with the length of $42 \mathrm{~mm}$, which is compared to that for the filament length of $55 \mathrm{~mm}$ under the experimental condition shown in Figure 5(a). This result indicates that modification in filament length will not change the Bessel beam pattern of the terahertz radiation but modify the divergence of this terahertz beam, resulting in a change in the ring number of this Bessel beam in a confined solid angle.

Second, we simulate the terahertz radiation from a $55 \mathrm{~mm}$ long superluminal filament with a uniform distribution in the velocity of the ionization front, i.e., $\alpha_{z}$ is not dependent on $z$ in the simulation. In this case, terahertz radiation from the filament behaves as a Cerenkov radiation, as in Figure 5(c). The wavefront of such radiation is in a plane (shown as the dashed line) with a particular radiation angle determined by the velocity of the ionization front. 
Meanwhile, the three-ring beam pattern and $\pi$-phase shift between neighbor rings is still obvious in the terahertz beam profile because the velocity of the ionization front remains superluminal.

Third, when the velocity of the ionization front is not superluminal, the beam pattern of the terahertz radiation from $55 \mathrm{~mm}$ long filament turns to a traditional conical emission, as shown in Figure 5(d). In this case, the velocity of the terahertz radiation from each plasma point source is faster than the velocity of the ionization front which dominates the emerging time of new plasma point sources. Hence, the wavefront overtaking of the terahertz radiation from different plasma sources no longer exists, leading to a conical terahertz radiation from a two-color plasma filament.

It is worthwhile to point out that the Bessel terahertz beam shows a higher energy concentration when comparing with the traditional conical terahertz beam. The inner ring of the Bessel terahertz beam carries over $68 \%(>1 / e)$ of the entire pulse energy with the angular divergence of about $5^{\circ}$, while the divergence of the traditional conical terahertz beam is normally $8 \sim 10^{\circ}$ [20]. For Bessel terahertz beams, higher energy concentration leads to good performance in propagation, collection, and beam profile maintenance. This shows great advantage for applications of such plasma terahertz sources.

\section{Conclusion}

In conclusion, it has been demonstrated that terahertz radiations with a Bessel beam profile can be generated from a superluminal two-color laser filament. This Bessel beam can be collected and detected in the far field. A theory model is presented, which can well describe the experimental observations, indicating that the Bessel terahertz beam comes from the wavefront overtaking (originated from the superluminal filament structure) and the constructive/ destructive interference of terahertz radiation from different plasma sources distributed along the superluminal two-color filament (originated from phase changing of the two-color laser components inside the plasma channel). This work shows for the first time the feasibility to achieve terahertz beam shaping by controlling a laser filament in air plasma and forms a foundation for promising terahertz sciences and applications.

\section{Data Availability}

The data that support the plots within this paper and other findings of this study are available from the corresponding author upon reasonable request.

\section{Conflicts of Interest}

The authors declare that there is no conflict of interest regarding the publication of this article.

\section{Authors' Contributions}

Z.Zhang and Y.Chen conceived the idea and designed the experiments. Z.Zhang, J.Zhang, T.Xia, L.Wang, and B.Han conducted the experiments. Z.Zhang and J.Zhang constructed the model and made the simulations. F.He and $Z$.Sheng gave the theoretical support of this project. Z.Zhang, Y.Chen, and Z. Sheng wrote the paper. Z.Sheng and Jie Zhang gave the general support of this project. Zhelin Zhang and Jiayang Zhang have contributed equally to this work.

\section{Acknowledgments}

This work is supported by the National Natural Science Foundation of China (11774228, 12074250, 11991073, 11721091, and 12135009) and Science and Technology Commission of Shanghai Municipality (16DZ2260200).

\section{Supplementary Materials}

A Calculation of the superluminal filament structure Figure 1: sketch diagram of the superlumial filament. Figure 2: measured terahertz waveforms emitted from $55 \mathrm{~mm}$ long superluminal filament. Figure 3: measured terahertz waveforms emitted from $42 \mathrm{~mm}$ long superluminal filament. Figure 4: (a) experimentally measured spatial distribution of the terahertz waveforms emitted from $42 \mathrm{~mm}$ long superluminal filament. (b) Simulated spatial distribution of terahertz waveforms compared with (a). (Supplementary Materials)

\section{References}

[1] D. McGloin and K. Dholakia, "Bessel beams: diffraction in a new light," Contemporary Physics, vol. 46, no. 1, pp. 15-28, 2005.

[2] J. Durnin, J. J. Miceli, and J. H. Eberly, "Diffraction-free beams," Physical Review Letters, vol. 58, no. 15, pp. 14991501, 1987.

[3] V. Garés-Chávez, D. McGloin, H. Melville, W. Sibbett, and K. Dholakia, "Simultaneous micromanipulation in multiple planes using a self- reconstructing light beam," Nature, vol. 419, no. 6903, pp. 145-147, 2002.

[4] C. Pfeiffer and A. Grbic, "Controlling vector bessel beams with metasurfaces," Physical Review Applied, vol. 2, no. 4, 2014.

[5] N. Bozinovic, Y. Yue, Y. Ren et al., "Terabit-scale orbital angular momentum mode division multiplexing in fibers," Science, vol. 340, no. 6140, pp. 1545-1548, 2013.

[6] J. Wang, J. Y. Yang, I. M. Fazal et al., “Terabit free-space data transmission employing orbital angular momentum multiplexing," Nature Photonics, vol. 6, no. 7, pp. 488-496, 2012.

[7] J. Ng, Z. Lin, and C. T. Chan, "Theory of optical trapping by an optical vortex beam," Physical Review Letters, vol. 104, no. 10, 2010.

[8] K. Toyoda, F. Takahashi, S. Takizawa et al., "Transfer of light helicity to nanostructures," Physical Review Letters, vol. 110, no. 14, 2013.

[9] F. Takahashi, K. Miyamoto, H. Hidai, K. Yamane, R. Morita, and T. Omatsu, "Picosecond optical vortex pulse illumination forms a monocrystalline silicon needle," Scientific Reports, vol. 6 , article 21738, 2016. 
[10] T. A. Planchon, L. Gao, D. E. Milkie et al., "Rapid threedimensional isotropic imaging of living cells using Bessel beam plane illumination," Nature Methods, vol. 8, no. 5, pp. 417423, 2011.

[11] A. Bitman, I. Moshe, and Z. Zalevsky, "Improving depth-of field in broadband $\mathrm{THz}$ beams using nondiffractive Bessel beams," Optics Letters, vol. 37, no. 19, pp. 4164-4166, 2012.

[12] G. Ok, S.-W. Choi, K. H. Park, and H. S. Chun, "Foreign object detection by sub-terahertz quasi-Bessel beam imaging," Sensors, vol. 13, no. 1, pp. 71-85, 2013.

[13] J. He, X. Wang, D. Hu et al., "Generation and evolution of the terahertz vortex beam," Optics Express, vol. 21, no. 17, pp. 20230-20239, 2013.

[14] X. Wei, C. Liu, L. Niu et al., "Generation of arbitrary order Bessel beams via $3 \mathrm{D}$ printed axicons at the terahertz frequency range," Applied Optics, vol. 54, no. 36, pp. 10641-10649, 2015.

[15] J. Kasparian, M. Rodriguez, G. Méjean et al., "White-light filaments for atmospheric analysis," Science, vol. 301, no. 5629, pp. 61-64, 2003.

[16] H. Li, E. Lötstedt, H. Li et al., "Giant enhancement of air lasing by complete population inversion in $N_{2}^{+}$," Physical Review Letters, vol. 125, article 053201, 2020.

[17] H. Zang, H. Li, W. Zhang et al., "Robust and ultralow-energythreshold ignition of a lean mixture by an ultrashort-pulsed laser in the filamentation regime," Light: Science \& Applications, vol. 10, no. 1, pp. 1-7, 2021.

[18] K. Y. Kim, A. J. Taylor, J. H. Glownia, and G. Rodriguez, "Coherent control of terahertz supercontinuum generation in ultrafast laser- gas interactions," Nature Photonics, vol. 2, no. 10, pp. 605-609, 2008.

[19] C. D'Amico, A. Houard, M. Franco et al., "Conical forward thz emission from femtosecond-laser-beam filamentation in air," Physical Review Letters, vol. 98, no. 23, 2007.

[20] Z. Zhang, Y. Chen, M. Chen et al., "Controllable terahertz radiation from a linear-dipole array formed by a two-color laser filament in air," Physical Review Letters, vol. 117, no. 24, 2016.

[21] Z. Zhang, Y. Chen, S. Cui et al., "Manipulation of polarizations for broadband terahertz waves emitted from laser plasma filaments," Nature Photonics, vol. 12, no. 9, pp. 554-559, 2018.

[22] M. Chen, A. Pukhov, X.-Y. Peng, and O. Willi, “Theoretical analysis and simulations of strong terahertz radiation from the interaction of ultrashort laser pulses with gases," Physical Review E, vol. 78, no. 4, 2008.

[23] V. A. Kostin and N. V. Vvedenskii, "Ionization-induced conversion of ultrashort Bessel beam to terahertz pulse," Optics Letters, vol. 35, pp. 247-249, 2010.

[24] H. Zhong, N. Karpowicz, and X.-C. Zhang, "Terahertz emission profile from laser-induced air plasma," Applied Physics Letters, vol. 88, no. 26, article 261103, 2006.

[25] Y. Lin, W. Seka, J. H. Eberly, H. Huang, and D. L. Brown, "Experimental investigation of Bessel beam characteristics," Applied Optics, vol. 31, no. 15, pp. 2708-2713, 1992.

[26] S. L. Chin, T. . J. Wang, C. Marceau et al., "Advances in intense femtosecond laser filamentation in air," Laser Phys, vol. 22, no. 1, pp. 1-53, 2012.

[27] Y. Wang, S. Yan, A. T. Friberg, D. Kuebel, and T. D. Visser, "Electromagnetic diffraction theory of refractive axicon lenses," Journal of the Optical Society of America. A, vol. 34, no. 7, pp. 1201-1211, 2017.
[28] R. Dharmavarapu, S. Bhattacharya, and S. Juodkazis, "Diffractive optics for axial intensity shaping of bessel beams," Journal of Optics, vol. 20, no. 8, article 085606, 2018.

[29] B. M. Penetrante and J. N. Bardsley, "Residual energy in plasmas produced by intense subpicosecond lasers," Physical Review A, vol. 43, no. 6, pp. 3100-3113, 1991.

[30] Z. Zhang, Y. Chen, Z. Zhang et al., "Phase evolution of terahertz radiation from femtosecond laser-induced air plasma," Optics Letters, vol. 45, no. 7, p. 1966, 2020. 